\title{
Blood Pressure Management in Patients with Type 2 Diabetes
}

\author{
Kazuo Eguchi
}

\begin{abstract}
In patients with type 2 diabetes (T2DM), the coexistence of hypertension enhances the cardiovascular risk, and the prevention of future cardiovascular disease is an important component of T2DM management. Antihypertensive therapy has been shown to be an effective method of reducing the micro- and macrovascular complications of T2DM, however, the optimal target blood pressure (BP) levels are still under debate. Most of the international guidelines have raised the target clinic BP from 130/80 to $140 / 90 \mathrm{mmHg}$, however, the Japanese Society of Hypertension 2014 guidelines kept the target BP level at 130/80 $\mathrm{mmHg}$. However, individualized BP-lowering treatments should be considered in patients with T2DM, especially in high-risk individuals such as those with a history of stroke or retinopathy, and aggressive antihypertensive therapy below $130 \mathrm{mmHg}$ should be initiated even when the initial systolic BP level is $<140 \mathrm{mmHg}$. The authors performed two studies concerning the BP target levels of home BP. In the first study, the authors found that a home BP target $<125 / 75 \mathrm{mmHg}$ was effective in improving the measures of vascular stiffness and kidney damage. In the second study, when the clinic BP target was set at 130/80 $\mathrm{mmHg}$, the home BP could be approximately $130 / 80 \mathrm{mmHg}$. More data are needed to individualize the target BP levels of T2DM patients.
\end{abstract}

Key words: type 2 diabetes, hypertension, target blood pressure level

(Intern Med 54: 2285-2289, 2015)

(DOI: 10.2169/internalmedicine.54.5617)

\section{Introduction}

In patients with type 2 diabetes (T2DM), coexisting hypertension enhances the cardiovascular risk, and appropriate antihypertensive treatment is necessary to prevent microand macrovascular complications. According to various observational and interventional studies, the clinic blood pressure (BP) value of $130 / 80 \mathrm{mmHg}$ was adopted as a target BP level (1-3). However, following the Action to Control Cardiovascular Risk in Diabetes (ACCORD) trial in 2010 (4), many of the international guidelines have raised the target clinic BP value to $140 / 90 \mathrm{mmHg}(5,6)$, and only a few guidelines have retained the target BP level of 130/80 $\mathrm{mmHg}(7,8)$.

Out-of-office BP monitoring, such as home BP (9-12) and ambulatory BP monitoring (13), has been shown to be useful in patients with T2DM. In the Japanese Society of Hypertension (JSH) 2014 guidelines (JSH 2014), the target home BP level was set at $125 / 75 \mathrm{mmHg}$ (7). To the best of my knowledge, there have been no data regarding the threshold of target home BP values in patients with diabetes. In this review, the author reassesses the target level of BP in patients with T2DM and discusses the management of hypertension in T2DM patients.

\section{Comparison of various international guidelines}

There are some differences in the target levels of clinic BP in patients with diabetes (Table). In the U.S. Seventh Report of the Joint National Committee on Prevention, Detection, Evaluation, and Treatment of High Blood Pressure (JNC-7) (1), the European Society of Hypertension (ESH) and European Society of Cardiology (ESC) guidelines (ESC/ ESH 2007) (2), and the JSH 2009 guidelines (3), the clinic BP target was set at $130 / 80 \mathrm{mmHg}$; however, in the current version of the ESH-ESC 2013 (5) and the American Diabetes Association guidelines (ADA 2013) (14), the clinic BP target was raised to $140 / 90 \mathrm{mmHg}$. Of note, in the ADA 2012 guidelines, the target was 130/80 $\mathrm{mmHg}$, but was al- 
Table. International Guidelines' Target BP Levels for Individuals with Type 2 Diabetes.

\begin{tabular}{llc}
\hline Organization & & Clinic BP (mmHg) \\
\hline JSH (Japan) & JSH 2009 & $<130 / 80$ \\
& JSH 2014 & $<130 / 80$ \\
JNC (U.S.) & JNC7 2003 & $<130 / 80$ \\
& JNC8 2014 & $<140 / 90$ \\
ADA (U.S.) & ADA 2012 & $<130 / 80$ \\
& ADA 2014 & SBP $<140 / 80$ \\
& & (young $<130)$ \\
ESH (Europe) & ESH-ESC2007 & $<130 / 80$ \\
& ESH-ESC2013 & $<140 / 85$ \\
& ESH/ESC Diabetes Guideline 2007 & $<130 / 80$ \\
& ESH/ESC Diabetes Guideline 2013 & $<140 / 85$ \\
NICE (England) & & $<140 / 80$, \\
& & but if retinopathy, \\
& & cerebrovascular \\
& NICE CG66 2008 & disease, or \\
& & microalbuminuria is \\
& & present, $<130 / 80$ \\
\hline
\end{tabular}

tered in 2013. On the other hand, in the UK's National Institute for Health and Clinical Excellence (NICE) guidelines (15), the target BP was $130 / 80 \mathrm{mmHg}$ in cases of retinopathy, history of stroke, and microalbuminuria, but was otherwise 140/80 mmHg. In the ADA 2013 guidelines, the target systolic blood pressure (SBP) value was set at $<140$ $\mathrm{mmHg}$, but was $<130 \mathrm{mmHg}$ in young subjects, whereas the target diastolic blood pressure (DBP) is $<80 \mathrm{mmHg}$ regardless of age (14).

In the ESH/ESC 2013 diabetes guidelines (ESH/ESC 2013), the target clinic BP was loosened to $140 / 85 \mathrm{mmHg}$ in patients with T2DM (16), however, a target SBP $<130$ $\mathrm{mmHg}$ was set for hypertensives with overt proteinuria and patients with diabetic nephropathy. This was based not only on the ACCORD trial findings but also on the "Reappraisal" which was published before the announcement of the ACCORD trial (17). Thus, a target BP level $<130 / 80 \mathrm{mmHg}$ in DM is not generally accepted, except under special conditions.

\section{The ACCORD trial}

The ACCORD trial published in 2010 is a very important study that has changed the way that BP is managed in patients with T2DM (4). Sponsored by the U.S. National Heart, Lung, and Blood Institute, the ACCORD trial investigated 4,733 patients with T2DM randomized to two different systolic BP targets $(120 \mathrm{mmHg}$ and $140 \mathrm{mmHg}$ ) and followed for 4.7 years. This trial revealed no significant differences between the groups in the primary endpoints of non-fatal myocardial infarction (MI), non-fatal stroke, and cardiovascular mortality. The only difference observed was a stroke endpoint as a secondary endpoint.

\section{Intensive BP lowering and stroke events}

The finding that aggressive BP lowering results in fewer stroke outcomes than standard therapy has been consistently demonstrated in meta-analyses. A meta-analysis which analyzed 13 clinical studies and a total of 1,330 subjects showed that a SBP of $130-135 \mathrm{mmHg}$ was appropriate in patients with T2DM or prediabetes (18). In comparison with the standard therapy group, the group with a target SBP $\leq 135 \mathrm{mmHg}$ showed a decrease in stroke events, especially in the group with SBP $\leq 130 \mathrm{mmHg}$. The rate of stroke events was also significantly reduced in the aggressive therapy group (19). Among certain ethnic groups, such as Asians, this result may be applicable in clinical practice (7). According to a very recent meta-analysis of T2DM (45 trials, 104,586 participants), aggressive antihypertensive therapy to achieve BP values below $130 \mathrm{mmHg}$ should be initiated in high-risk individuals, such as those with a history of stroke and retinopathy, even when the initial SBP level is $<140 \mathrm{mmHg}(20)$.

\section{Effectiveness of intensive BP lowering}

There are several studies and meta-analyses which showed no benefit of intensive BP lowering on cardiovascular outcomes. In the aforementioned meta-analysis, the rates of other endpoints, including total mortality, cardiovascular death, MI, and heart failure (HF), were similar to those of the standard therapy group (18). Another meta-analysis concluded that aggressive BP lowering in subjects with T2DM did not significantly reduce the rates of mortality and incident MI (19). In that report, five clinical trials (ABCD-H, ABCD-N, HOT, ABCD-2V, and ACCORD-BP) were included in the analyses, and DBP (except for in the ACCORD trial) was a target of BP-lowering therapy.

\section{Safety of intensive BP lowering}

There have been several reports which showed adverse effects of intensive BP-lowering therapy. In the aforementioned meta-analysis, the rate of serious adverse events was $20 \%$ higher in the intensive treatment group compared to the standard therapy group (of note, serious adverse events were increased by $40 \%$ in the group with SBP $\leq 130$ mmHg) (18). In the Irbesartan Diabetic Nephropathy Trial, patients who achieved $\mathrm{BP} \leq 120 / 85 \mathrm{mmHg}$ had an increased incidence of cardiovascular events (21).

In a retrospective analysis of the United Kingdom General Practice Research Database (22), the achieved clinic BP was used to classify the patients as the aggressive control group $(<130 / 80 \mathrm{mmHg}$ ), the standard control group (130 to $<140 /$ 80 to $<85 \mathrm{mmHg}$ ), or the poor control group ( $\geq 140 \mathrm{mmHg}$, $\geq 85 \mathrm{mmHg}$ ), and the authors analyzed the cardiovascular outcomes. In diabetic patients with a history of cardiovascular disease (CVD), those with clinic BP $<110 / 75 \mathrm{mmHg}$ had increased mortality. On the other hand, among diabetic patients without a history of CVD, the risk was increased when the clinic BP was $<120 / 75 \mathrm{mmHg}$. The possibility of excessive BP lowering in T2DM should be monitored.

\section{Intensive BP lowering and microvascular disease}

In patients with T2DM, microvascular disease itself was demonstrated to be associated with CVD and stroke (23). In a sub-analysis of the ACCORD trial, the progression of ret- 
(a)

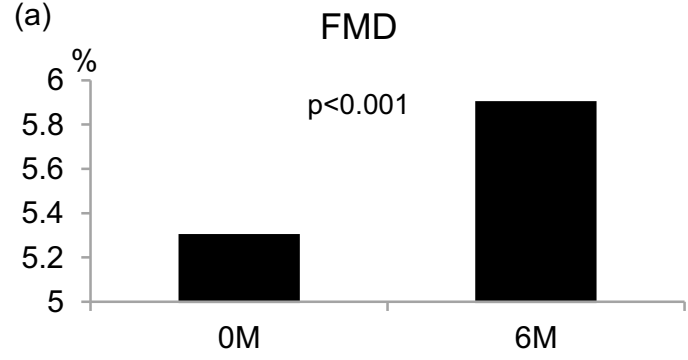

(b) $\quad$ Al adjusted by HR 75

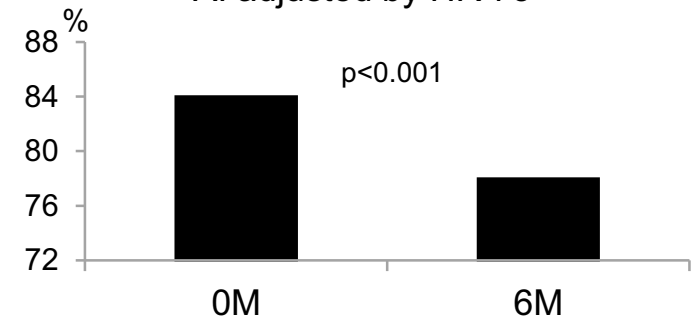

(c)
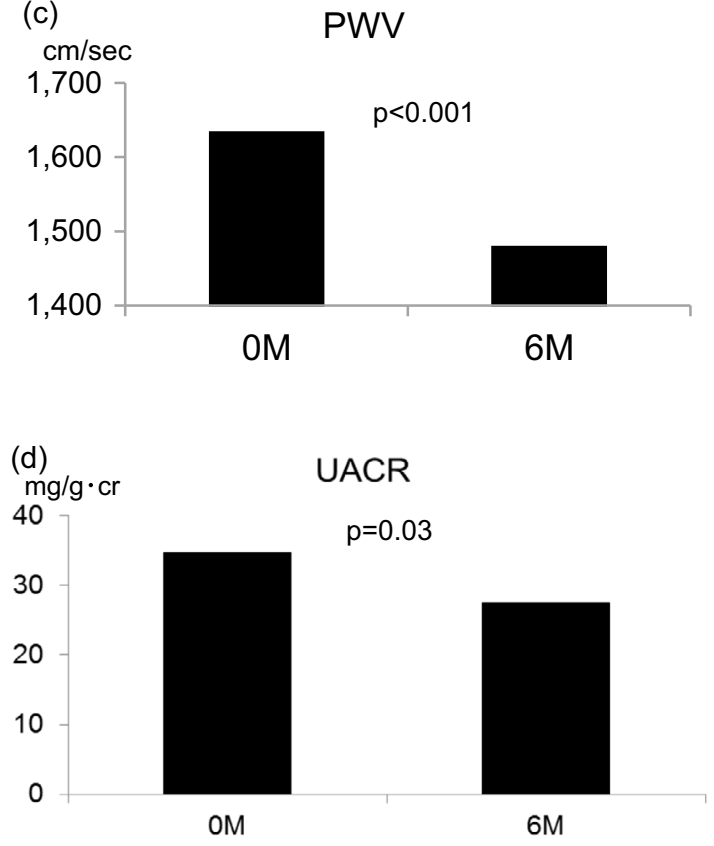

Figure 1. Measures of target organ damage at baseline (0M) and 6 months $(6 \mathrm{M})$ among 59 patients with uncomplicated diabetes or prediabetes. Changes in the flow-mediated vasodilation (FMD) (a), radial augmentation index (AI) adjusted by a heart rate (HR) of 75 beats/min (b), pulse wave velocity (PWV) (c), and the urinary albumin excretion ratio (UACR) as the geometric mean (d) (adapted from ref. 11).

inopathy was associated with future CVD (24). Namely, microvascular disease caused by hypertension and DM can present a risk of macrovascular disease, thus early intervention is clinically very important.

Aggressive BP control can improve the microvascular outcomes, such as retinopathy, nephropathy, and neuropathy (25). This effect was confirmed even in normotensive diabetes patients (26). The UKPDS 36 study showed that therapy lowering the T2DM patients' BP values by an aver- age of SBP $10 \mathrm{mmHg}$ reduced the risk of retinopathy by $13 \%(\mathrm{p}<0.001)$ and nephropathy by $13 \%(\mathrm{p}<0.001)(27)$. In the ACCORD trial, macroalbuminuria was improved by the aggressive BP treatment, however, a decrease in the estimated glomerular filtration rate (eGFR) and an increase of creatinine $(\mathrm{Cr})$ were rather prevalent in this group compared to the standard treatment group. In the aforementioned metaanalysis, the intensive treatment group (defined as those that achieved SBP $\leq 135 \mathrm{mmHg}$ ) showed a reduction in newonset microalbuminuria by $17 \%$ and a $27 \%$ lower risk of nephropathy. The group with SBP $<130 \mathrm{mmHg}$ showed a $36 \%$ lower risk of nephropathy (18). Therefore, with regard to the prevention of microvascular disease, lower target BP levels should therefore be considered.

\section{Effectiveness of a home BP target $<125 / 75 \mathrm{mmHg}$}

For individualized treatment, out-of-office BP, especially home $\mathrm{BP}$, is a convenient and effective method in clinical practice (7). In the JSH 2014 guidelines, the home BP target for patients with diabetes was set at $<125 / 75 \mathrm{mmHg}$ (7). In 2012 we performed a prospective study to aggressively lower the BP to a target home BP $<125 / 75 \mathrm{mmHg}$ in patients with uncomplicated diabetes or prediabetes $(n=59)$ (mean age: $62.6 \pm 9.4$ years, $51.7 \%$ men) (11). The patients' home morning SBP values were $>135 \mathrm{mmHg}$ at the time of enrollment. The target home BP was $<125 / 75 \mathrm{mmHg}$, and ARB (irbesartan), CCB (amlodipine), and/or a thiazide mimic (indapamide) were added according to the protocol. In addition to the assessment of BP, flow-mediated vasodilation (FMD) as a measure of the endothelial function, pulse wave velocity (PWV), radial augmentation index (AI), baroreflex sensitivity (BRS), and urine microalbumin were examined before and after the treatment. The results demonstrated that the patients' clinic SBP values fell significantly from $147 \pm 18$ to $125 \pm 15 \mathrm{mmHg}(\mathrm{p}<0.001)$, whereas their clinic DBP values did not change significantly (from $83 \pm 12$ to $80 \pm 54 \mathrm{mmHg}$ ). On the other hand, the patients' home SBP values fell significantly from $145 \pm 17$ to $128 \pm 11$ $\mathrm{mmHg}$, and their home DBP fell significantly from $81 \pm 11$ to $73 \pm 9 \mathrm{mmHg}$ (both $\mathrm{p}<0.001$ ). The FMD, PWV, and radial AI values (as measures of target organ damage) changed from $5.3 \pm 2.1 \%$ to $5.9 \pm 1.6 \%, 1,635 \pm 252 \mathrm{~cm} / \mathrm{s}$ to $1,472 \pm 184$ $\mathrm{cm} / \mathrm{s}$, and $84 \pm 10 \%$ to $78 \pm 11 \%$, respectively (Fig. 1).

In the same study (11), $22 \%$ of the patients experienced at least one study-related adverse effects: dizziness in seven, peripheral edema in four, and impotence in one patient. There were no serious adverse events that required study cessation. Although not all the patients achieved the target home BP level, aggressive BP-lowering therapy in hypertensive patients with diabetes or prediabetes may be effective in improving the measures of target organ damage, and may therefore be a good strategy for the relatively early stage of diabetes. 

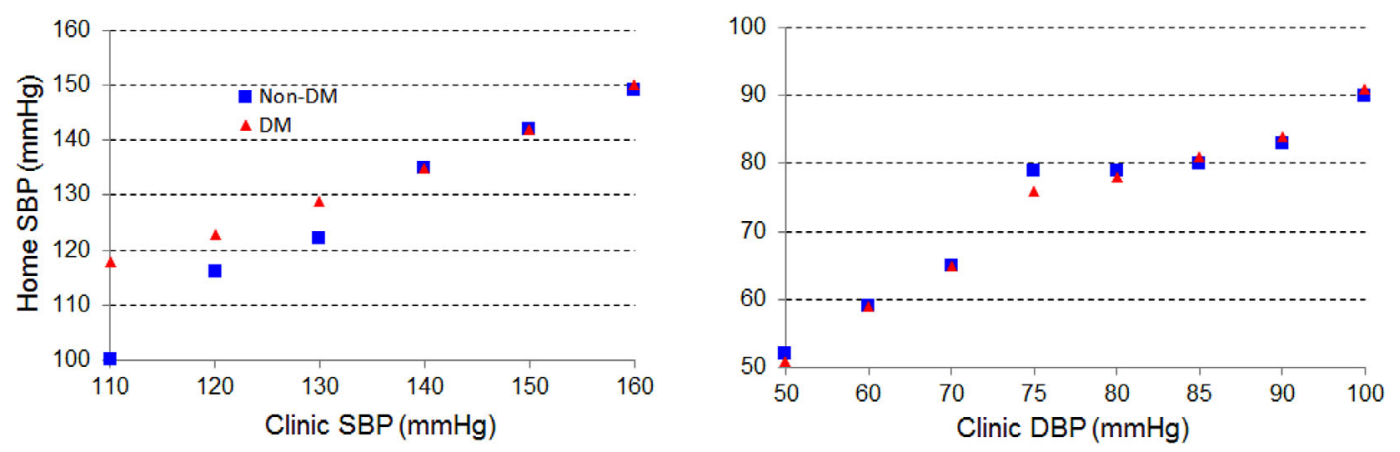

Figure 2. The home SBP and DBP levels corresponded to the clinic SBP and DBP levels in both the DM and non-DM patients. Because UACR values were used to connect the clinic and home SBP/DBP levels, the UACR data do not appear in the Figure (adapted from ref. 28).

\section{New proposed target home BP in subjects with T2DM}

The optimal target value for home BP in patients with T2 DM has not been established. We previously performed a cross-sectional study to explore the target home BP level in T2DM patients as a sub-analysis of the Japan Morning Surge Home Blood Pressure (J-HOP) Study (28). The JHOP study is a prospective observational study evaluating the predictive values of home BP for cardiovascular events in Japanese subjects with any cardiovascular risk fac-

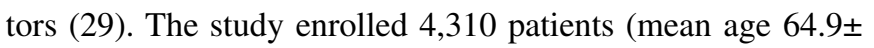
10.9 years; $47.0 \%$ men), and clinic and home BP monitoring were performed. The urinary albumin creatinine ratio (UACR) was used as a marker of microvascular disease. Quadratic equations of the relationship between the clinic/ home SBP and log-transformed UACR were used to determine the home BP value.

Home BP levels corresponding to clinic BP levels using the UACR values were calculated separately by the presence/absence of diabetes. The study included 1,057 (24.5\%) subjects with T2DM (the DM group) and 3,253 (75.5\%) without T2DM (the non-DM group). As shown in Fig. 2, the home BP levels equivalent to a clinic BP 140/90 $\mathrm{mmHg}$ were $135 / 84$ and $135 / 83 \mathrm{mmHg}$ in the $\mathrm{DM}$ and non-DM groups, respectively. The home SBP levels equivalent to a clinic SBP $130 / 80 \mathrm{mmHg}$ were $122 / 79 \mathrm{mmHg}$ in the nonDM group and $129 / 78 \mathrm{mmHg}$ in the DM group. Regardless of the diabetic status, the home BP level corresponding to the clinic SBP $140 / 90 \mathrm{mmHg}$ was $135 / 85 \mathrm{mmHg}$. In patients with T2DM, the home SBP level equivalent to a clinic SBP $130 / 80 \mathrm{mmHg}$ was $129 / 78 \mathrm{mmHg}$ with regard to the extent of microvascular disease. This issue must be confirmed in further prospective studies, however, these new thresholds may be appropriate in relation to previous reports.

In summary, the risks and benefits of aggressive antihypertensive treatment in patients with T2DM are:

Risks of aggressive treatment: increase in total mortality by too much BP lowering, increase in serious adverse events, and impaired organ blood flow (e.g., myocardial ischemia due to coronary flow).

Benefits of aggressive treatment: reduction of stroke incidence, prevention of microvascular disease, and improvement of the measures of target organ damage (e.g., microalbuminuria, endothelial function, pulse wave velocity).

In conclusion, in patients with T2DM, the concept of "the lower the better" has already been discarded. A target BP level $<130 / 80 \mathrm{mmHg}$ is limited to special populations, such as those covered by the JSH 2014 guidelines. However, the target BP level can vary depending on what disease is the focus of prevention. Therefore, individualized BP targets should be considered in patients with T2DM.

The author states that he has no Conflict of Interest (COI).

\section{References}

1. Chobanian AV, Bakris GL, Black HR, et al. Seventh Report of the Joint National Committee on Prevention, Detection, Evaluation, and Treatment of High Blood Pressure. Hypertension 42: 12061252, 2003.

2. Mancia G, De Backer G, Dominiczak A, et al. 2007 Guidelines for the management of arterial hypertension: the Task Force for the Management of Arterial Hypertension of the European Society of Hypertension (ESH) and of the European Society of Cardiology (ESC). Eur Heart J 28: 1462-1536, 2007.

3. Ogihara T, Kikuchi K, Matsuoka H, et al. The Japanese Society of Hypertension Guidelines for the Management of Hypertension (JSH 2009). Hypertens Res 32: 3-107, 2009.

4. ACCORD Study Group, Cushman WC, Evans GW, et al. Effects of intensive blood-pressure control in type 2 diabetes mellitus. $\mathrm{N}$ Engl J Med 362: 1575-1585, 2010.

5. Mancia G, Fagard R, Narkiewicz K, et al. 2013 ESH/ESC Guidelines for the management of arterial hypertension: The Task Force for the Management of Arterial Hypertension of the European Society of Hypertension (ESH) and of the European Society of Cardiology (ESC). Eur Heart J 34: 2159-2219, 2013.

6. James PA, Oparil S, Carter BL, et al. 2014 evidence-based guideline for the management of high blood pressure in adults: report from the panel members appointed to the Eighth Joint National Committee (JNC 8). JAMA 311: 507-520, 2013.

7. Shimamoto K, Ando K, Fujita T, et al. The Japanese Society of Hypertension Guidelines for the Management of Hypertension (JSH 2014). Hypertens Res 37: 253-390, 2014.

8. Dasgupta K, Quinn RR, Zarnke KB, et al. The 2014 Canadian 
Hypertension Education Program Recommendations for Blood Pressure Measurement, Diagnosis, Assessment of Risk, Prevention, and Treatment of Hypertension. Can J Cardiol 30: 485-501, 2014.

9. Kamoi K, Miyakoshi M, Soda S, Kaneko S, Nakagawa O. Usefulness of home blood pressure measurement in the morning in type 2 diabetic patients. Diabetes Care 25: 2218-2223, 2002.

10. Eguchi K, Matsui Y, Shibasaki S, et al. Controlling evening BP as well as morning BP is important in hypertensive patients with prediabetes/diabetes: the JMS-1 study. Am J Hypertens 23: 522-527, 2010.

11. Eguchi K, Hoshide S, Ishikawa S, Shimada K, Kario K. Aggressive blood pressure-lowering therapy guided by home blood pressure monitoring improves target organ damage in hypertensive patients with type 2 diabetes/prediabetes. J Clin Hypertens 14: 422428, 2012.

12. Noguchi Y, Asayama K, Staessen JA, et al. Predictive power of home blood pressure and clinic blood pressure in hypertensive patients with impaired glucose metabolism and diabetes. J Hypertens 31: 1593-1602, 2013.

13. Eguchi K, Pickering TG, Hoshide S, et al. Ambulatory blood pressure is a better marker than clinic blood pressure in predicting cardiovascular events in patients with/without type 2 diabetes. Am J Hypertens 21: 443-450, 2008.

14. American Diabetes Association. Standards of Medical Care in Diabetes - 2013. Diabetes Care 36: S11-S66, 2013.

15. Hypertension: The Clinical Management of Primary Hypertension in Adults. Update of Clinical Guidelines 18 and 34. [Internet]. National Clinical Guideline Centre, Ed. Commissioned by the National Institute for Health and Clinical Excellence (NICE), Royal College of Physicians (UK), London, 2011. [cited 2013 Jan. 4]. Available from: http://www.nice.org.uk/guidance/cg127/documents/ hypertension-update-full-guideline2

16. Authors/Task Force Members, Rydén L, Grant PJ, et al. ESC Guidelines on diabetes, pre-diabetes, and cardiovascular diseases developed in collaboration with the EASD: The Task Force on diabetes, pre-diabetes, and cardiovascular diseases of the European Society of Cardiology (ESC) and developed in collaboration with the European Association for the Study of Diabetes (EASD). Eur Heart J 34: 3035-3087, 2013.

17. Mancia G, Laurent S, Agabiti-Rosei E, et al. Reappraisal of European guidelines on hypertension management: a European Society of Hypertension Task Force document. J Hypertens 27: 2121$2158,2009$.
18. Bangalore S, Kumar S, Lobach I, Messerli FH. Blood pressure targets in subjects with type 2 diabetes mellitus/impaired fasting glucose: observations from traditional and bayesian random-effects meta-analyses of randomized trials. Circulation 123: 2799-2810, 2011.

19. McBrien K, Rabi DM, Campbell N, et al. Intensive and standard blood pressure targets in patients with type 2 diabetes mellitus: systematic review and meta-analysis. Arch Intern Med 172: 12961303, 2012.

20. Emdin CA, Rahimi K, Neal B, Callender T, Perkovic V, Patel A. Blood pressure lowering in type 2 diabetes: a systematic review and meta-analysis. JAMA 313: 603-615, 2015.

21. Berl T, Hunsicker LG, Lewis JB, et al. Impact of achieved blood pressure on cardiovascular outcomes in the Irbesartan Diabetic Nephropathy Trial. J Am Soc Nephrol 16: 2170-2179, 2005.

22. Vamos EP, Harris M, Millett C, et al. Association of systolic and diastolic blood pressure and all cause mortality in people with newly diagnosed type 2 diabetes: retrospective cohort study. BMJ 345: e5567, 2012.

23. Fuller JH, Stevens LK, Wang SL. Risk factors for cardiovascular mortality and morbidity: the WHO Mutinational Study of Vascular Disease in Diabetes. Diabetologia 44: S54-S64, 2001.

24. Gerstein HC, Ambrosius WT, Danis R, et al. Diabetic retinopathy, its progression, and incident cardiovascular events in the ACCORD trial. Diabetes Care 36: 1266-1271, 2013.

25. Lv J, Neal B, Ehteshami P, et al. Effects of intensive blood pressure lowering on cardiovascular and renal outcomes: a systematic review and meta-analysis. PLoS Med 9: e1001293, 2012.

26. Mehler PS, Coll JR, Estacio R, Esler A, Schrier RW, Hiatt WR. Intensive blood pressure control reduces the risk of cardiovascular events in patients with peripheral arterial disease and type 2 diabetes. Circulation 107: 753-756, 2003.

27. Adler AI, Stratton IM, Neil HAW, et al. Association of systolic blood pressure with macrovascular and microvascular complications of type 2 diabetes (UKPDS 36): prospective observational study. BMJ 321: 412-419, 2000.

28. Eguchi K, Hoshide S, Komori T, Nagasaka S, Kario K. A new proposal for the target value for home BP in type 2 diabetes patients: the J-HOP study. Am J Hypertens 28: 971-979, 2015.

29. Hoshide S, Kario K, Yano Y, et al. Association of morning and evening blood pressure at home with asymptomatic organ damage in the J-HOP study. Am J Hypertens 27: 939-947, 2014.

(C) 2015 The Japanese Society of Internal Medicine http://www.naika.or.jp/imonline/index.html 